\title{
Inventaire des coproduits agricoles potentiellement utilisables pour la production de pupes de mouche Hermetia illucens ( $L$. 1758) pour l'alimentation piscicole au Bénin
}

\author{
Armel Gougbedji, Pamphile Agbohessou, Philippe Adédjobi Lalèyè, Fréderic Francis \& Rudy \\ Caparros Megido
}

Armel Gougbedji : Béninois, Doctorant, Université de Liège, Gembloux Agro-Bio Tech, Unité d’Entomologie Fonctionnelle et Évolutive, 5030 Gembloux, Belgique. Email :

gougbemel@yahoo.fr, Tel : +32 4655758 07/+229 97603437

Pamphile Agbohessou : Béninois, Doctorant, Université de Namur, Faculté des sciences, Unité de Recherche en Biologie Environnementale et Évolutive, BE-5000, Belgique. Email :

agbohessou.pamphile@yahoo.fr.

Philippe Adédjobi Lalèyè : Béninois, PhD, Professeur titulaire au CAMES, Doyen, Université d'Abomey-Calavi, Faculté des Sciences Agronomiques, Laboratoire d'Hydrobiologie et d’Aquaculture, 01 BP 526 Cotonou, Bénin. Email : laleyephilippe@gmail.com

Fréderic Francis : Belge, PhD, Professeur ordinaire, Doyen, Université de Liège, Gembloux AgroBio Tech, Unité d'Entomologie Fonctionnelle et Évolutive, 5030 Gembloux, Belgique. Email : frederic.francis@uliege.be

Rudy Caparros Megido : Belge, PhD, Premier assistant, Université de Liège, Gembloux Agro-Bio Tech, Unité d’Entomologie Fonctionnelle et Évolutive, 5030 Gembloux, Belgique. Email :

r.caparros@uliege.be

reçu le 13 mai 2020 et accepté le 5 août 2020

DOI: $10.25518 / 2295-8010.1587$

Résumé :

Cette recherche vise l'inventaire et la sélection de coproduits agricoles d'intérêt pour l'élevage d'Hermetia illucens (L. 1758) au Bénin. Les données relatives à la nature, la disponibilité et la valeur marchande des coproduits agricoles ont été collectées à travers une enquête dans 8 départements du Bénin. Deux régimes expérimentaux isoprotéiques et isolipidiques ont été formulés à partir de trois coproduits principaux (tourteau de coton, son de maïs et abats de poisson) et testés sur la croissance larvaire et la qualité nutritionnelle des prépupes d' $H$. illucens. 28 coproduits agricoles ont été recensés et répartis en 3 groupes : les tourteaux, les résidus et les sons. Le substrat à base de son de maïs et d'abats de poisson favorise une croissance optimale des larves. Les teneurs en protéines $(47,89 \pm 0,11 \%)$ et en lipides $(26,75 \pm$ $0,29 \%$ ) des prépupes issues de ce régime permettent de répondre aux besoins nutritionnels du tilapia du Nil. Le régime à base de tourteau de coton a induit une forte mortalité $(67,33 \pm 3,96$ $\%)$ chez les larves d'H. illucens. Sur la base des facteurs de disponibilité, de compétition et des résultats de l'expérimentation, 22 coproduits agricoles sont retenus pour de futurs essais. 
Inventaire des coproduits agricoles potentiellement utilisables pour la produ...

\section{Abstract : \\ Inventory of agricultural by-products potentially usable for the production of Hermetia illucens (L. 1758) as fish feed in Benin}

This research aims to identify and select agricultural by-products of interest for the breeding of Hermetia illucens (L. 1758) in Benin. Data on the nature, availability and market value of agricultural by-products were collected through a survey in 8 departments of Benin. Two experimental isoprotein and isolipid diets were formulated from the three main by-products (cotton cake, maize bran, fish offal) and tested on larval growth and nutritional quality of $H$. illucens prepupae. 28 agricultural by-products were identified and divided into 3 groups: oilcake, residues and bran. The substrate based on maize bran and fish offal showed optimal larval growth. Protein (47.89 $\pm 0.11 \%)$ and lipid (26.75 $\pm 0.29 \%)$ contents of prepupae from this diet meet the nutritional requirements of the Nile tilapia. The cotton meal diet induced high mortality $(67.33 \pm 3.96 \%)$ in $H$. illucens larvae. On the basis of availability and competition factors and the results of the experiment, 22 agricultural co-products were selected for further assays on $H$. illucens rearing.

\section{Introduction}

La production halieutique au Bénin repose essentiellement sur la pêche continentale (80\% des captures) et ne permet de couvrir que $35 \%$ des besoins d'une population en constante croissance (8). Le poisson, bien qu'étant une source d'apport protéique majeure ne contribue qu'à 28,5\% des besoins de la population béninoise. Au regard des derniers travaux d'estimation des stocks réalisés, il est indéniable que les ressources ichtyques des écosystèmes continentaux connaissent une baisse alarmante $(3,14,15)$. Dans un souci de sécurité alimentaire et de sauvegarde des stocks naturels, les perspectives se concentrent désormais sur l'aquaculture. Ainsi plusieurs projets, à l'instar du Projet de Vulgarisation de l'Aquaculture Continentale en République du Bénin (PROVAC) sont venus soutenir les initiatives de pisciculture (25). À travers ces programmes, les élevages du poisson, Clarias gariepinus (Burchell 1822)* et du tilapia du Nil, Oreochromis niloticus* (L. 1758) ont connu une forte expansion. En raison des préférences alimentaires, le tilapia du Nil est devenu l'espèce principalement élevée pour le marché local. L'essor de cette filière reste cependant contraint à plusieurs facteurs dont la disponibilité d'aliments locaux performants pour le poisson et peu onéreux (27). En effet, les aliments utilisés en pisciculture au Bénin tels que le Coppens, le Skretting, le Raanan sont importés et s'avèrent très couteux (29). De plus, les efforts de formulation d'aliment à partir d'ingrédients locaux ont connu des résultats mitigés, principalement en terme de croissance des poissons $(10,27)$. L'un des enjeux majeurs dans la composition d'aliments pour poisson est la disponibilité de ressources présentant des taux de protéines élevés et des profils lipidiques adéquats. Le coût de la farine de poisson habituellement utilisée comme source protéique dans les formules alimentaires influence fortement le développement de la pisciculture. Par ailleurs, la surpêche d'espèces de poissons sauvages à l'instar de la sardine commune ou des anchois qui sont ensuite transformés en farine protéique représente un impact négatif important sur les écosystèmes vu le rôle important de ces espèces dans les réseaux trophiques aquatiques (9). En quête de solutions alternatives, plusieurs expérimentations ont testé l'incorporation des insectes dans les régimes alimentaires des poissons d'élevage (12). Les insectes, capables de convertir efficacement les matières organiques en biomasses de haute valeur protéique, représentent de parfaits candidats à la substitution de la farine de poisson. Les espèces telles que Tenebrio molitor 
Linné, 1758, Oryctes rhinoceros (L. 1758), Bombyx mori L. 1758, Musca domestica L. 1758 et Hermetia illucens (L. 1758) ont déjà été testés dans l'alimentation de différentes espèces de poissons $(16,12)$.

Une tendance se dégage de plus en plus pour le choix de la mouche-soldat noire, Hermetia illucens (Diptère, Stratiomyidae). Outre leurs aptitudes reconnues pour la gestion de grands volumes de déchets $(4,7)$, cette espèce détritivore possède un large spectre alimentaire et se nourrit préférentiellement de résidus organiques humides (2). Ainsi, la mouche est souvent élevée sur des substrats composés de coproduits agricoles ou résidus de transformations agroalimentaires (22, 23). Les expériences antérieurs de nutrition ont utilisé les aliments pour poulet « Chicken feed » (19), des déchets végétaux et de restaurant $(22,31)$, des mélasses de betterave, des épluchures de pomme de terre, des céréales, de la levure de bière, des restes de pain et de biscuits (22), de la farine de luzerne, du son de blé, de la farine de maïs, de la drèche de brasserie (33) et des abats de poissons (32). Dès lors, l'élevage de $H$. illucens exige une connaissance des matières disponibles pour constituer son substrat de vie. C'est à cette fin que le présent travail a été effectué et dont l'objectif est de recenser les coproduits agricoles hypothétiquement utilisables pour produire les larves de la mouche-soldat noire. Au-delà d'un inventaire brut, la finalité est de sélectionner les ressources sur la base de leur disponibilité et de leur potentiel nutritif.

\section{Matériel et Méthode}

\section{Zone d'étude}

La présente étude a été conduite sur la base d'une enquête systématique auprès de deux types de structures alimentaires : les provenderies et les établissements de transformation agroalimentaire. En prenant comme critère la représentativité de ces deux pôles, l'enquête a été réalisée dans 8 départements du Bénin (Fig.1) : Atlantique, Littoral, Mono, Couffo, Zou, Collines, Ouémé et Borgou. Les données ont été collectées dans 48 structures réparties dans les différentes communes de chaque département (Figure 1). 


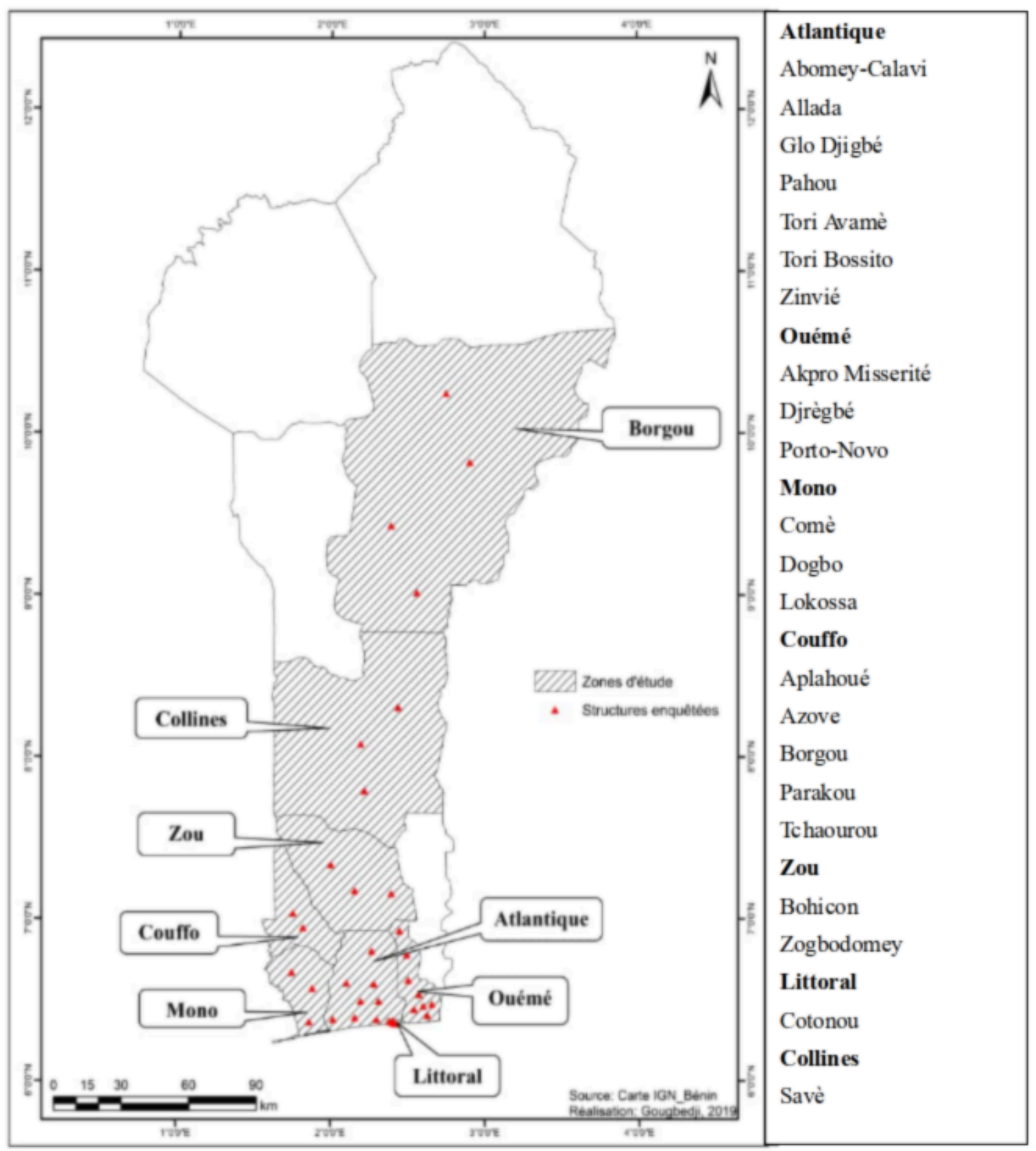

Figure 1 : Les communes de chaque département sont présentées à droite de la figure

\section{Inventaire et sélection des coproduits agricoles pour l'élevage d'Hermetia illucens}

Une recherche préliminaire a permis d'obtenir auprès du Centre de Commerce International du Bénin (CCIB) et de la Direction de l'Alimentation et de la Nutrition Appliquée (DANA), une liste des acteurs des deux secteurs enquêtés. Un guide d'entretien a été conçu puis testé au préalable avant l'enquête définitive. Les entretiens semi-directifs effectués ont abordé la nature, la disponibilité, l'abondance et le coût d'approvisionnement des coproduits identifiés. La nature reprend les différentes catégories de coproduits agricoles notamment les tourteaux, les sons et les résidus de 
transformation agro-alimentaire. La disponibilité des matières a été évaluée suivant les possibilités d'approvisionnement sur un cycle annuel. L'abondance des produits a été estimée sur la base des quantités mobilisables par mois. Les prix de vente minimum et maximum sur chaque produit ont été enregistrés afin d'en déterminer les coûts moyens.

Les coproduits pouvant servir de substrats nourriciers aux larves d'insectes ont été choisis sur la base de leur disponibilité dans le temps. Seul les coproduits disponibles toute l'année ont été sélectionnés.

\section{Croissance des larves d'Hermetia illucens nourries avec des coproduits agricoles}

Des larves de poids moyens 0,01 g ont été utilisées pour cette expérience. Elle proviennent d'une reproduction des adultes de mouche-soldat noire effectuée à l'insectarium du Laboratoire d'Hydrobiologie et d'Aquaculture de l'Université d'Abomey-Calavi. Le dispositif expérimental était constitué de 9 bacs plastiques ( 42 x 29 x $25 \mathrm{~cm}$ ) obtenus dans le marché local « Dantokpa ». Chaque bac est muni d'une pente pour faciliter la sortie et la récolte des prépupes à la fin de l'expérience (Fig.2).

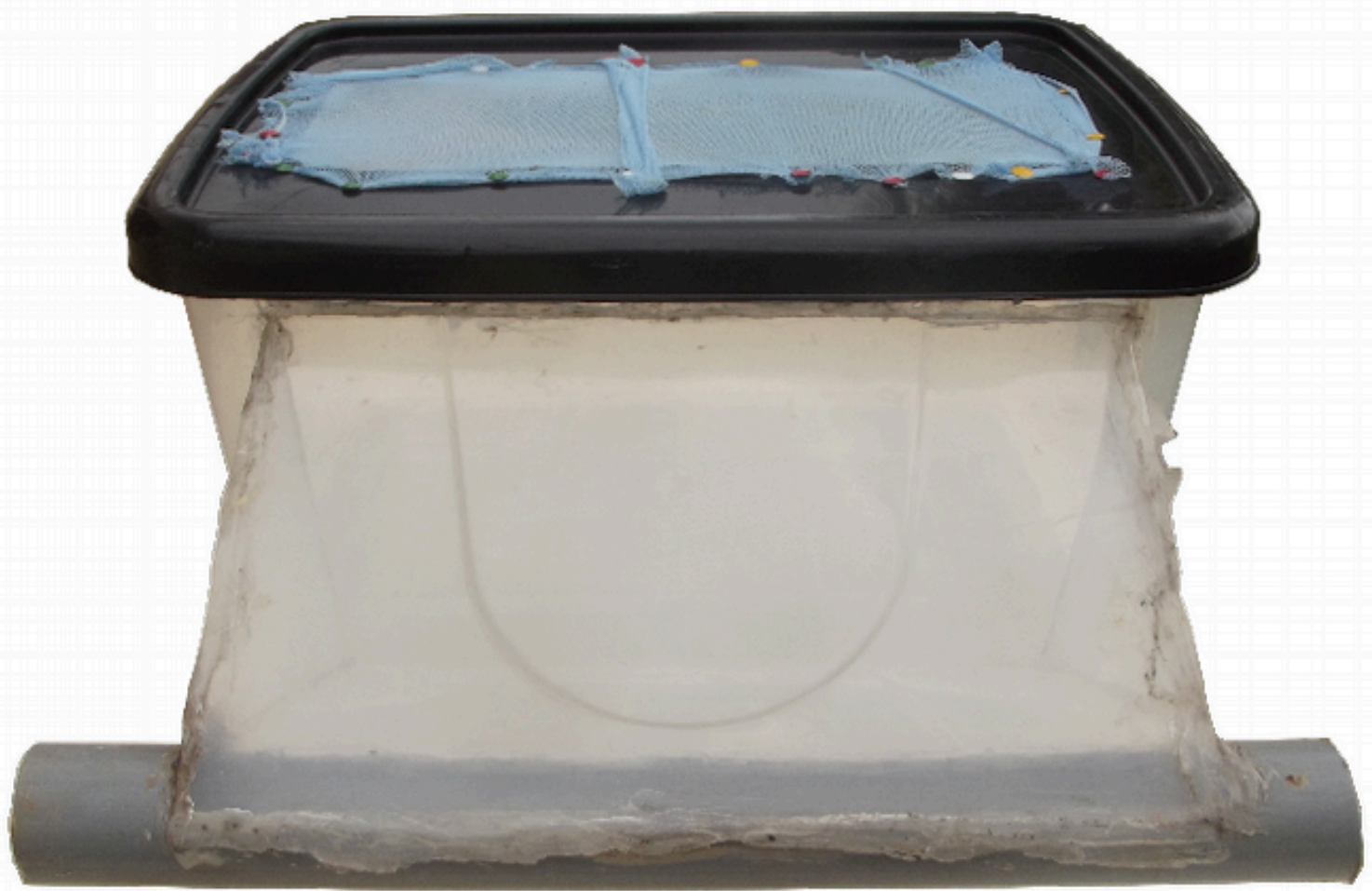

Figure 2 : Dispositif d'élevage larvaire

Afin de composer les substrats expérimentaux, un coproduit a été choisi par catégorie. Ainsi deux régimes isoprotéiques et isolipidiques ont été formulés et testés en triplicat à partir du son de maïs, du tourteau de coton et d'abats de poisson (Tableau 1). Le régime témoin était de la provende pour poulet obtenu chez le groupe Véto Service au Bénin. Cet aliment est classiquement utilisé comme 
Inventaire des coproduits agricoles potentiellement utilisables pour la produ...

contrôle dans la littérature (Nguyen et al., 2013; Spranghers et al., 2017). Chaque coproduit a été broyé en poudre dans un moulin électrique (David $4 \mathrm{~V}$, Italie). Dans chaque bac, $2 \mathrm{~kg}$ de matière sèche de chaque régime on été introduits et humidifiés à $70 \%$. La densité de charge larvaire a été de 2 larves/g de substrat.

Tableau 1 : Composition des différents substrats nourriciers.

\begin{tabular}{ccccc}
\hline Régimes & Coproduits & $\begin{array}{c}\text { Proportion } \\
(\%)\end{array}$ & Protéine (\%) & Lipide (\%) \\
\hline R0 & Provende & 100 & 21,00 & 5,60 \\
R1 & Son de maïs & 77 & 22,48 & 5,38 \\
& Abats de poisson & 23 & & \\
R2 & Son de maïs & 60 & 22,50 & 5,38 \\
& Tourteau de coton & 40 & &
\end{tabular}

Tous les 2 jours, 30 larves ont été échantillonnées dans chaque bac et pesées avec une balance électronique (Sartorius, FB423, Allemagne). Les mortalités ont été également relevées dans les différentes structures d'élevage. L'expérience de croissance a pris fin lorsque les premières prépupes ont émergé du substrat. Les substrats résiduels ont été séchés dans une étuve électrique (Heraeus, Lille, France) à $105^{\circ} \mathrm{C}$ pendant $3 \mathrm{~h}$ puis pesés avec une balance électronique (Ohaus Scout Pro, SPU20001, USA).

\section{Composition nutritionnelle des prépupes d'Hermetia illucens}

Toutes les prépupes ont été récoltées des différents bacs, nettoyées à l'eau puis congelées pendant $24 \mathrm{~h}$. Elles ont ensuite été séchées dans une étuve électrique à $60^{\circ} \mathrm{C}$ pendant 6 heures puis broyées. Des analyses de la matière sèche, des cendres, des protéines brutes et des lipides totaux ont été réalisées sur les différentes farines d'insecte. Les teneurs en matière sèche ont été déterminées par déshydratation des échantillons à l'aide d'une étuve à $105^{\circ} \mathrm{C}$ pendant $1 \mathrm{~h}$. Les teneurs en cendres ont été déterminées en incinérant les échantillons dans un four à $550^{\circ} \mathrm{C}$ à une vitesse constante de $50^{\circ} \mathrm{C}$ toutes les $30 \mathrm{~min}$ pendant $4 \mathrm{~h}$, puis refroidies dans un dessiccateur. Les teneurs en protéines brutes ont été déterminées par la méthode «Dumas» en utilisant un facteur de conversion des protéines en azote de 4,76 chez les insectes (13). Les teneurs en matières grasses ont été déterminées par la méthode d'extraction de « Folch » telle que décrite par Paul et al. (24).

\section{Analyse des données}

Afin d'appréhender la diversité des coproduits agricoles par département ainsi que leur spécificité, les indices de diversité de Shannon H' (Eq.1) et d'équitabilité de Piélou E (Eq.2) ont été calculés. 


$$
\text { (1) } H=-\sum_{i=1}^{s} P i \log _{2} P i
$$

Avec $\mathrm{Pi}=\mathrm{Ni} / \mathrm{N}$ où Ni représente l'effectif du coproduit agricole i et $\mathrm{N}$ l'effectif total des coproduits.

$$
\text { (2) } E=H^{\prime} / \log _{2} S
$$

Le taux de décomposition des substrats (DS) par les larves a été calculé pour chaque régime (Eq.3).

$$
\text { (3) } D S=(S I-S F) \times 100 / S I
$$

Avec SI la quantité de substrat initiale et SF la quantité de substrat final après décomposition.

L'analyse de variance à 1 critère (F) et la somme des rangs de Kruskal-Wallis (H) ont été effectuées pour comparer les variables entre elles. Ces tests ont été réalisés après vérification des conditions d'application d'une analyse de la variance. Lorsque les différences étaient significatives, le test de Mann-Whitney était réalisé pour comparer les variables deux à deux. Toutes les analyses ont été réalisées avec le logiciel $\mathrm{R} 3.03$.

\section{Résultats}

\section{Description de la population étudiée}

La population ciblée dans le cadre de cette étude est composée de structures réparties majoritairement dans le sud et le centre du Bénin. L'effectif total des coproduits agricoles identifiés est constitué de 176 observations. La figure 3 présente l'importance par ordre décroissant d'observation des coproduits agricoles par département. Les départements de l'Ouémé, de l'Atlantique et du Borgou concentrent l'essentiel des coproduits en matière d'abondance (76,70 \%). Parmi les communes, celle de Porto-Novo possède le plus grand nombre d'observation suivite de celles de Parakou et d'Abomey-Calavi (Fig. 4). 


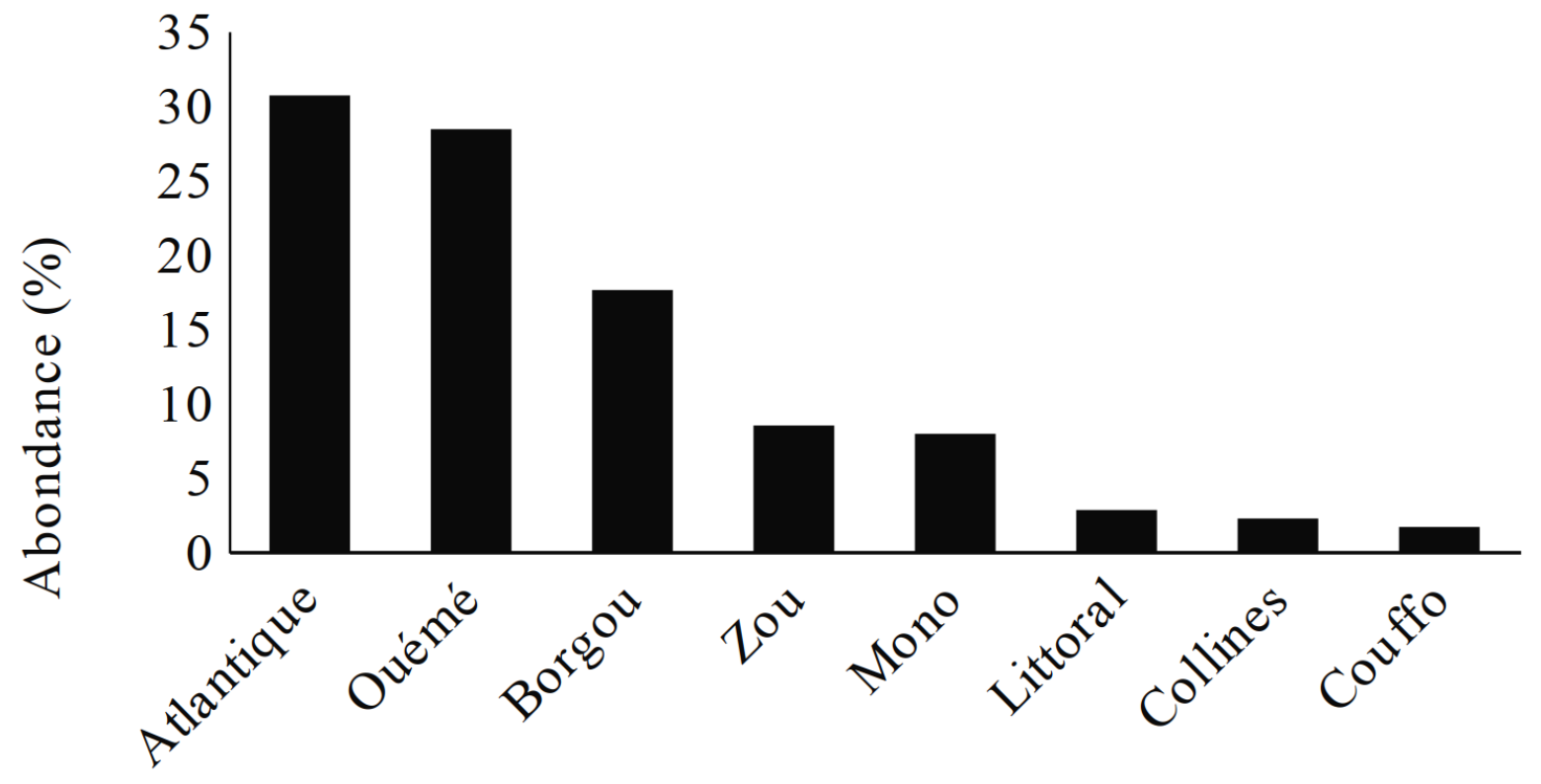

Département

Figure 3 : Histogramme d'abondance des coproduits agricoles par département.

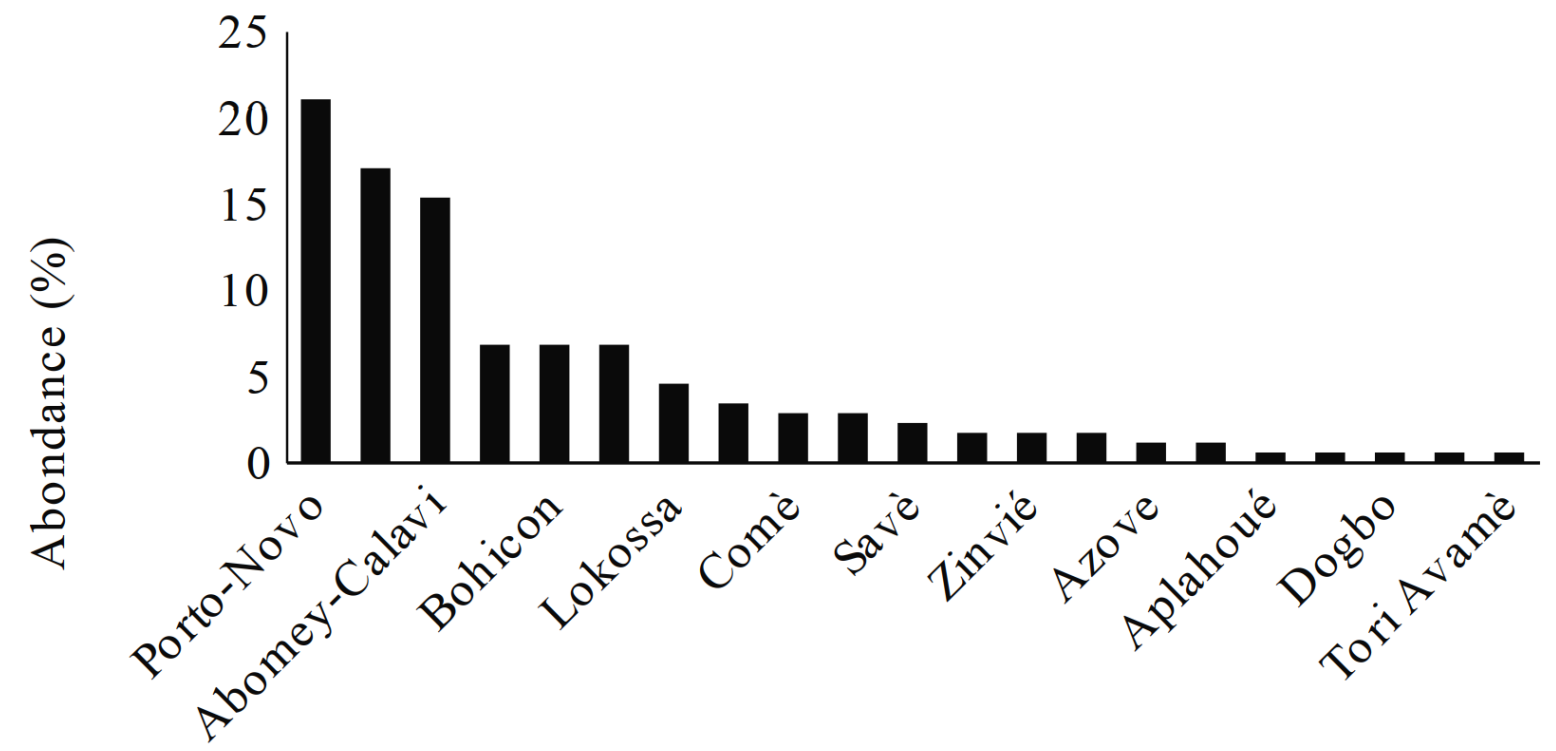

Commune

Figure 4 : Histogramme d'abondance des coproduits agricoles par commune.

La figure 5 présente la répartition des observations au sein des 3 groupes de coproduits identifiés. Les tourteaux sont les plus représentés suivis des sons et des résidus de transformation agroalimentaire. 


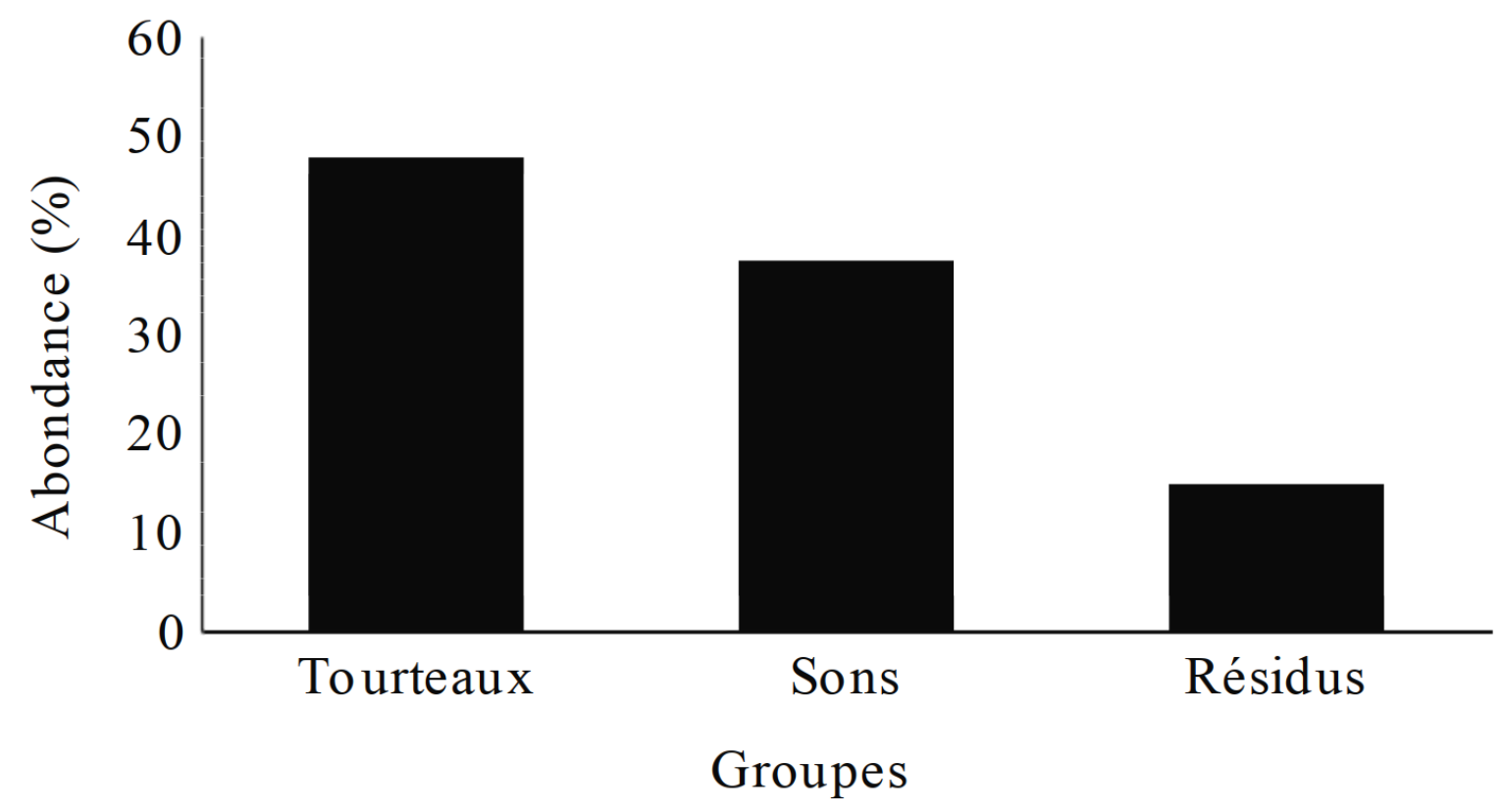

Figure 5 : Représentation de l'abondance des coproduits agricoles par groupe.

\section{Diversité et distribution géographique des coproduits agricoles}

Le tableau 2 présente le répertoire des coproduits agricoles inventoriés. Au total, 28 coproduits agricoles ont été identifiés sur l'ensemble de la zone d'étude. Certains départements ont le monopole de production de certains coproduits agricoles. Les tourteaux de tournesol, de moringa, de baobab, le son de sorgho, la farine de néré et la farine de baobab se retrouvent essentiellement dans le département du Borgou. Les épluchures de manioc, les épluchures d'igname et la farine de blé avariée sont uniquement représentés dans le département de l'Atlantique. Les résidus de fromage et de mangue sont essentiellement produits dans les collines. Le Zou garde l'exclusivité du tourteau d'acajou et de la farine d'acajou. Les abats de poisson et les résidus de gingembre sont retrouvés dans le département du littoral. Les brisures de pain et les peaux de banane sont recensées dans l'Ouémé.

Les valeurs des indices de diversité (Tableau 1) de Shannon-Wiener $\left(\mathrm{H}^{\prime}=0,74\right)$ et d'équitabilité de Piélou $(E=0,81)$ dénotent d'une forte diversité et d'une équirépartition des coproduits de façon globale à travers les départements.

Tableau 2 : Profil général de la diversité des coproduits agricoles inventoriés. Les chiffres du tableau représentent les structures dans lesquelles les coproduits sont retrouvés. 


\begin{tabular}{|c|c|c|c|c|c|c|c|c|c|}
\hline Coproduits agricoles & $\begin{array}{c}\text { Atlantiqu } \\
\text { e }\end{array}$ & $\begin{array}{c}\text { Borgo } \\
\text { u } \\
\end{array}$ & $\begin{array}{c}\text { Colline } \\
\mathrm{s}\end{array}$ & $\begin{array}{c}\text { Couff } \\
\text { o } \\
\end{array}$ & $\begin{array}{c}\text { Littora } \\
1 \\
\end{array}$ & $\begin{array}{c}\text { Mon } \\
0 \\
\end{array}$ & $\begin{array}{c}\text { Ouém } \\
\text { é }\end{array}$ & Zou & $\begin{array}{c}\text { Tota } \\
1 \\
\end{array}$ \\
\hline Abats de poisson & 0 & 0 & 0 & 0 & 1 & 0 & 0 & 0 & 1 \\
\hline Cossette de manioc & 0 & 1 & 0 & 0 & 0 & 0 & 1 & 1 & 3 \\
\hline Drèche de brasserie & 2 & 1 & 0 & 0 & 0 & 0 & 0 & 0 & 3 \\
\hline Drèche de soja & 1 & 0 & 0 & 0 & 0 & 0 & 0 & 1 & 2 \\
\hline Epluchures de manioc & 2 & 0 & 0 & 0 & 0 & 0 & 0 & 0 & 2 \\
\hline Epluchures d'igname & 1 & 0 & 0 & 0 & 0 & 0 & 0 & 0 & 1 \\
\hline Farine d'acajou & 0 & 0 & 0 & 0 & 0 & 0 & 0 & 1 & 1 \\
\hline Farine de baobab & 0 & 1 & 0 & 0 & 0 & 0 & 0 & 0 & 1 \\
\hline Farine de blé avariée & 1 & 0 & 0 & 0 & 0 & 0 & 0 & 0 & 1 \\
\hline Farine de néré & 0 & 1 & 0 & 0 & 0 & 0 & 0 & 0 & 1 \\
\hline Résidus d'acajou & 0 & 2 & 1 & 0 & 1 & 0 & 0 & 0 & 4 \\
\hline Résidus d'ananas & 0 & 0 & 1 & 0 & 1 & 0 & 1 & 0 & 3 \\
\hline Résidus de fromage & 0 & 0 & 1 & 0 & 0 & 0 & 0 & 0 & 1 \\
\hline Résidus de gingembre & 0 & 0 & 0 & 0 & 1 & 0 & 0 & 0 & 1 \\
\hline Résidus de mangue & 0 & 0 & 1 & 0 & 0 & 0 & 0 & 0 & 1 \\
\hline Son de blé & 10 & 4 & 0 & 0 & 0 & 3 & 8 & 1 & 26 \\
\hline Son de maïs & 6 & 2 & 0 & 0 & 1 & 0 & 7 & 2 & 18 \\
\hline Son de riz & 6 & 2 & 0 & 0 & 0 & 5 & 7 & 1 & 21 \\
\hline Son de sorgho & 0 & 1 & 0 & 0 & 0 & 0 & 0 & 0 & 1 \\
\hline Tourteau d'acajou & 0 & 0 & 0 & 0 & 0 & 0 & 0 & 1 & 1 \\
\hline Tourteau d'arachide & 0 & 1 & 0 & 1 & 0 & 0 & 0 & 0 & 2 \\
\hline Tourteau de baobab & 0 & 2 & 0 & 0 & 0 & 0 & 0 & 0 & 2 \\
\hline Tourteau de coton & 8 & 4 & 0 & 0 & 0 & 2 & 9 & 3 & 26 \\
\hline Tourteau de moringa & 0 & 1 & 0 & 0 & 0 & 0 & 0 & 0 & 1 \\
\hline Tourteau de palmiste & 9 & 4 & 0 & 2 & 0 & 3 & 8 & 1 & 27 \\
\hline Tourteau de soja & 8 & 2 & 0 & 0 & 0 & 1 & 9 & 3 & 23 \\
\hline Tourteau de souchet & 0 & 1 & 0 & 0 & 0 & 0 & 0 & 0 & 1 \\
\hline
\end{tabular}

\begin{tabular}{|c|c|c|c|c|c|c|c|c|c|}
\hline Coproduits agricoles & $\begin{array}{c}\text { Atlantiqu } \\
\text { e }\end{array}$ & $\begin{array}{c}\text { Borgo } \\
\text { u }\end{array}$ & $\begin{array}{c}\text { Colline } \\
s\end{array}$ & $\begin{array}{c}\text { Couff } \\
\text { o }\end{array}$ & $\begin{array}{c}\text { Littora } \\
1\end{array}$ & $\begin{array}{c}\text { Mon } \\
\text { o }\end{array}$ & $\begin{array}{c}\text { Ouém } \\
\text { é }\end{array}$ & Zou & $\begin{array}{c}\text { Tota } \\
1\end{array}$ \\
\hline Tourteau de tournesol & 0 & 1 & 0 & 0 & 0 & 0 & 0 & 0 & 1 \\
\hline Total global & 54 & 31 & 4 & 3 & 5 & 14 & 50 & 15 & 176 \\
\hline Abondance proportionnelle (Pi) & 0,31 & 0,18 & 0,02 & 0,02 & 0,03 & 0,08 & 0,28 & $\begin{array}{c}0,0 \\
9\end{array}$ & 1 \\
\hline Indice de Shannon $\left(\mathrm{H}^{\prime}\right)$ & & & & & & & & & 0,74 \\
\hline Indice d'équitabilité de Piélou (E) & & & & & & & & & $\mathbf{0 , 8 1}$ \\
\hline$H^{\prime} \max$ & & & & & & & & & $\mathbf{0 , 9 0}$ \\
\hline
\end{tabular}

Disponibilité temporelle, quantitative et valeur marchande des coproduits agricoles identifiés

\section{Disponibilité temporelle, quantitative et valeur marchande des coproduits agricoles identifiés}

La plupart des coproduits inventoriés ont une disponibilité annuelle, à la fois en saison sèche et en saison pluvieuse (Tableau 3). A l'exception de la farine de baobab, des épluchures d'igname, des résidus de mangue, de la farine de blé avariée et du son de sorgho qui ne peuvent être obtenues qu'à des périodes précises de l'année, les autres ne sont contraints à aucune limite temporelle.

Le tableau 3 présente également les quantités et les coûts des coproduits. Les coproduits du groupe des tourteaux sont les plus produits $(120,15 \pm 659,69 \mathrm{t} / \mathrm{mois})$ suivis de ceux des résidus $(32,66 \pm 77,85 \mathrm{t} / \mathrm{mois})$ et des sons $(19,12 \pm 34,23 \mathrm{t} / \mathrm{mois})\left(\mathrm{H}_{2}=6,34 ; p=0,04\right)$. La dispersion des quantités entre les structures est très élevée pour la plupart des coproduits agricoles. Parmi les tourteaux, ceux du soja et du coton présentent les quantités les plus élevées, à savoir 95,63 \% des 
quantités cumulées. Au sein des résidus, la drèche de brasserie et les résidus d'ananas sont les plus abondants (92,46 \% des quantités cumulées) et seul le son de sorgho se retrouve en faible quantité dans le groupe des sons $(0,04 \%)$.

Des trois catégories de coproduits, les tourteaux coutent le plus cher et les résidus sont les moins cher de tous $\left(\mathrm{H}_{2}=59,87 ; p<0,001\right)$. Parmi les résidus, les abats de poisson, les épluchures d'igname et de manioc sont obtenues gratuitement. Le coproduit le plus couteux est le tourteau de moringa (1000 FCFA/Kg). Les autres coproduits varient de 25 FCFA/Kg à 425 FCFA/Kg.

Tableau 3 : Disponibilité temporelle, quantité et coût des coproduits agricoles

\begin{tabular}{|c|c|c|c|c|c|c|c|}
\hline \multirow{2}{*}{ Groupe } & \multirow{2}{*}{ Coproduits } & \multirow{2}{*}{ Disponibilité } & \multirow{2}{*}{$\mathbf{N}$} & \multicolumn{2}{|c|}{ Quantités (t/mois) } & \multicolumn{2}{|c|}{ Coûts (FCFA/Kg) } \\
\hline & & & & Min-Max & Moyenne \pm écart-type & Min-Max & Moyenne \pm écart-type \\
\hline \multirow{6}{*}{ Tourteaux } & Tourteau de palmiste & Annuelle & 27 & $0,2-20$ & \multirow{6}{*}{$120,15 \pm 659,69^{\mathrm{a}}$} & $100-190$ & \multirow{6}{*}{$231,88 \pm 142,35^{\mathrm{a}}$} \\
\hline & Tourteau de coton & Annuelle & 26 & $1-100$ & & $110-240$ & \\
\hline & Tourteau d'arachide & Annuelle & 2 & $2,4-30$ & & $400-450$ & \\
\hline & Tourteau de baobab & Annuelle & 2 & $15-30$ & & $100-150$ & \\
\hline & Tourteau de moringa & Annuelle & 1 & 5 & & 1000 & \\
\hline & Tourteau de souchet & Annuelle & 1 & 2 & & 100 & \\
\hline \multirow{11}{*}{ Résidus } & Résidus d'acajou & Annuelle & 4 & $1,5-2,5$ & \multirow{11}{*}{$32,66 \pm 77,85^{\mathrm{b}}$} & $85-160$ & \multirow{11}{*}{$95,19 \pm 72,71^{\mathrm{b}}$} \\
\hline & Drèche de brasserie & Annuelle & 3 & $75-315$ & & $40-45$ & \\
\hline & Résidus d'ananas & Annuelle & 3 & $10-100$ & & $150-300$ & \\
\hline & Cossette de manioc & Annuelle & 3 & $1-20$ & & $100-155$ & \\
\hline & Farine de néré & Annuelle & 1 & 5 & & 110 & \\
\hline & Résidus de gingembre & Annuelle & 1 & 5 & & 150 & \\
\hline & Abats de poisson & Annuelle & 1 & 2 & & 0 & \\
\hline & Farine d'acajou & Annuelle & 1 & 1,5 & & 75 & \\
\hline & Farine de blé avariée & Rare & 1 & 0,25 & & 85 & \\
\hline & Résidus de mangue & Saison pluvieuse & 1 & 0,25 & & 200 & \\
\hline & Résidus de fromage & Annuelle & 1 & 0,15 & & 25 & \\
\hline \multirow{4}{*}{ Sons } & Son de blé & Annuelle & 26 & $0,5-100$ & \multirow{4}{*}{$19,12 \pm 34,23^{c}$} & $100-190$ & \multirow{4}{*}{$113,09 \pm 31,55^{c}$} \\
\hline & Son de riz & Annuelle & 21 & $1-85$ & & $40-110$ & \\
\hline & Son de maïs & Annuelle & 18 & $0,05-208$ & & $100-150$ & \\
\hline & Son de sorgho & Rare & 1 & 0,5 & & 100 & \\
\hline
\end{tabular}

\section{Paramètres d'élevage des larves et composition nutritionnelle des prépupes d'Hermetia illucens}

Le poids moyen final des prépupes produites (Tableau 4) sur des résidus est significativement inférieur 0,13 \pm 0,00 g pour R1 (son de maïs + abats de poisson) et 0,14 \pm 0,01 g pour R2 (son de maïs + tourteau de coton) à celui du témoin R0 0,18 \pm 0,00 g (provende). Les larves ont atteint un pic de croissance avant de connaître une perte de poids progressive jusqu'au stade prépupal. Les larves du régime contrôle ont mis 10 jours pour atteindre la phase de prépupe alors que celles des régimes $\mathrm{R} 1$ et $\mathrm{R} 2$ ont mis respectivement 12 et 16 jours (Fig.6). 


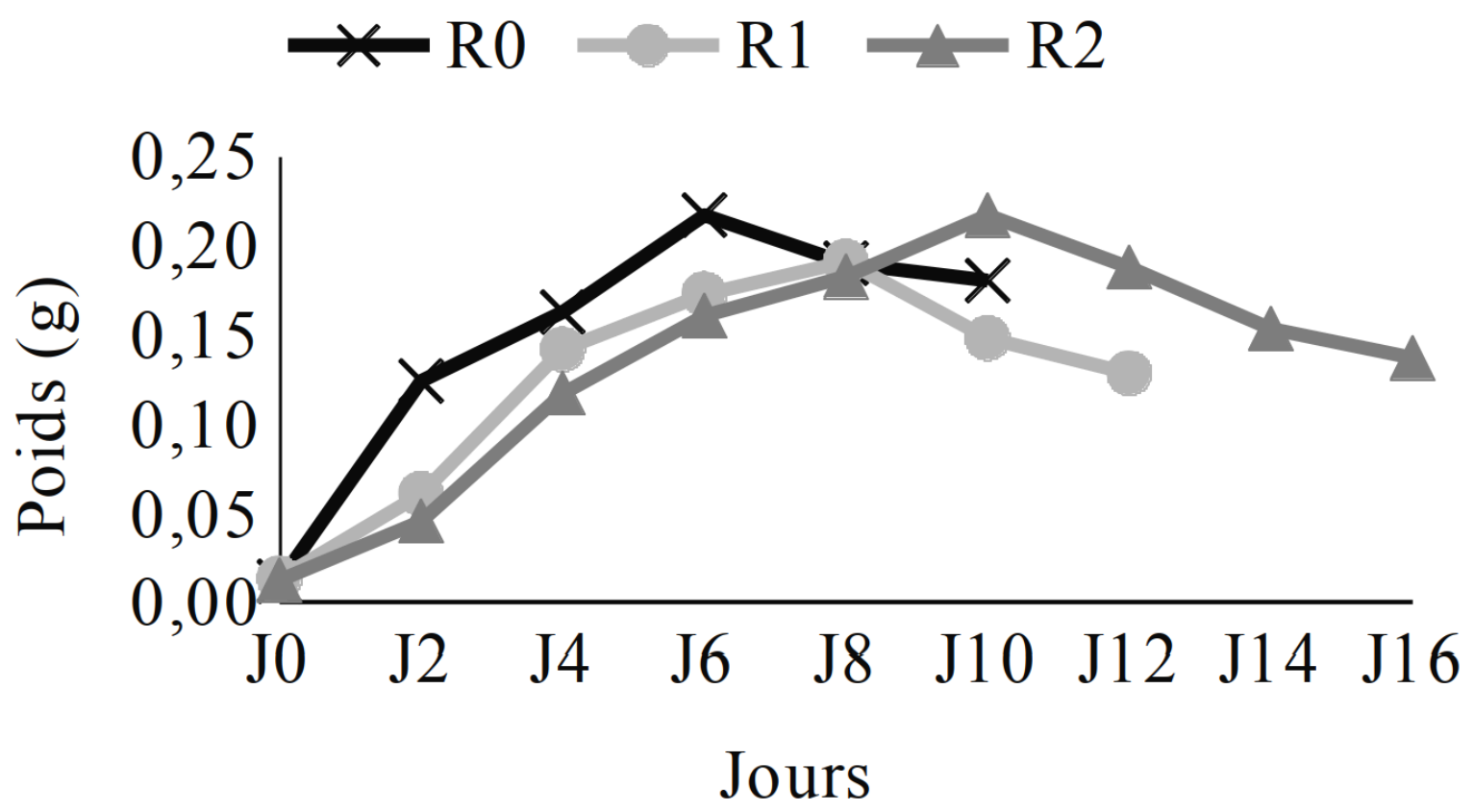

Figure 6 : Évolution journalière du poids moyen des larves d'Hermetia illucens en fonction des régimes alimentaires.

Des trois régimes testés, seules les populations nourries avec le son de maïs et le tourteau de coton ont enregistré une forte mortalité (67,33 \pm 3,96 \%) (Tableau 4). De même, le plus faible taux de décomposition du substrat a été enregistré avec ce régime $(37,85 \% \pm 0,88 \%)\left(F_{2,6}=5271 ; p\right.$ $<0,001)$.

Le taux de matière sèche le plus élevé est obtenu avec le régime témoin $(84,56 \pm 0,50 \%)$ alors que le plus faible est enregistré chez les prépupes du régime R1 (80,43 $\pm 0,30 \%$; Tableau 4). De même, les prépupes du contrôle ont les teneurs les plus élevées en cendre $(10,87 \pm 0,07 \%)$. Les prépupes nourries avec le son de maïs et les abats de poisson ont les teneurs les plus élevées en protéines $(47,89 \pm 0,11 \%)$ tandis que les prépupes du régime témoin présentent les plus faibles valeurs $(43,39 \pm 0,10 \%)$. En ce qui concerne les lipides, les taux les plus élevés sont extraits des prépupes nourries avec le son de maïs et le tourteau de coton $(27,70 \pm 0,11 \%)$ et les plus faibles chez celles du témoin $(22,65 \pm 0,17 \%)$.

Tableau 4 : Paramètres biologiques et nutritionnels de larves et prépupes d'Hermetia illucens. 


\begin{tabular}{|c|c|c|c|c|c|c|}
\hline & & R0 & R1 & $\mathbf{R 2}$ & $\begin{array}{c}\text { Test } \\
\text { statistique } \\
\end{array}$ & $\mathbf{p}$ \\
\hline \multirow{4}{*}{$\begin{array}{l}\text { Paramètres } \\
\text { biologiques }\end{array}$} & Poids prépupes & & & & $\mathrm{F}_{2,6}=102,73$ & $<0,001$ \\
\hline & (g) & $0,18 \pm 0,00^{\mathrm{a}}$ & $0,13 \pm 0,00^{\mathrm{b}}$ & $0,14 \pm 0,01^{\mathrm{b}}$ & \multirow{3}{*}{$\begin{array}{c}\mathrm{F}_{2,6}=865,73 \\
\mathrm{~F}_{2,6}=5271\end{array}$} & \multirow{3}{*}{$\begin{array}{l}<0,001 \\
<0,001\end{array}$} \\
\hline & Mortalité (\%) & $0,00 \pm 0,00^{\mathrm{a}}$ & $0,00 \pm 0,00^{\mathrm{a}}$ & $67,33 \pm 3,96^{\mathrm{b}}$ & & \\
\hline & DS & $81,66 \pm 0,31^{a}$ & $74,82 \pm 0,30^{\mathrm{b}}$ & $37,85 \pm 0,88^{c}$ & & \\
\hline \multirow{5}{*}{$\begin{array}{l}\text { Paramètres } \\
\text { nutritionnels }\end{array}$} & Matière sèche & & & & $F_{2,6}=211,51$ & $<0,001$ \\
\hline & $(\%)$ & $84,56 \pm 0,50^{a}$ & $80,43 \pm 0,30^{b}$ & $82,47 \pm 0,15^{c}$ & \multirow{4}{*}{$\begin{array}{l}F_{2,6}=33,74 \\
F_{2,6}=3189 \\
F_{2,6}=1031\end{array}$} & \multirow{4}{*}{$\begin{array}{l}<0,001 \\
<0,001 \\
<0,001\end{array}$} \\
\hline & Cendres (\%) & $10,87 \pm 0,07^{\mathrm{a}}$ & $5,99 \pm 0,01^{b}$ & $5,30 \pm 0,03^{\mathrm{c}}$ & & \\
\hline & Protéines (\%) & $43,39 \pm 0,10^{\mathrm{a}}$ & $47,89 \pm 0,11^{\mathrm{b}}$ & $47,10 \pm 0,11^{c}$ & & \\
\hline & Lipides (\%) & $22,65 \pm 0,17^{\mathrm{a}}$ & $26,75 \pm 0,29^{b}$ & $27,70 \pm 0,11^{\mathrm{c}}$ & & \\
\hline
\end{tabular}

Les différentes lettres présentent des résultats significativement différents

\section{Discussion}

Cette étude prospective a permis d'identifier une diversité de coproduits agricoles susceptibles de servir de substrats nourriciers aux larves de $H$. illucens. L'inventaire a recensé 28 coproduits agricoles qui intègrent plusieurs substrats d'usages (ou associés) en élevage larvaire chez cette espèce. Outre les résidus d'acajou, d'ananas, de fromage et de gingembre ; les drèches de brasserie et de soja ; les abats de poisson, qui constituent des matières humides, les autres coproduits sont de nature sèche. L'utilisation de ces résidus secs pour l'élevage devrait donc être conditionnée par une humidification. En effet, les larves de la mouche-soldat noire ne se développent que dans les milieux dont le taux d'humidité est de l'ordre de 60-70\% (28). Au-delà de l'humidité, certains auteurs ont rapporté que la texture du substrat influençait fortement la vitesse de croissance et le taux de survie des larves de $H$. illucens $(11,19)$. Ainsi les coproduits agricoles non farineux ou extrêmement fibreux doivent être homogénéisés afin d’optimiser l'élevage larvaire.

Les coproduits inventoriés se constituent en trois groupes : les tourteaux qui sont les plus produits, les résidus, suivis des sons. Bien que leur régularité soit élevée à travers la zone d'étude, certains coproduits sont spécifiques à certains départements. Le département du Borgou monopolise les tourteaux de tournesol, de souchet, de moringa, de baobab, le son de sorgho, la farine de néré et la farine de baobab. Les épluchures de manioc, les épluchures d'igname et la farine de blé avariée sont retrouvés dans le département de l'Atlantique. Les résidus de fromage et de mangue sont produits dans les Collines. Le tourteau et la farine d'acajou ont été recensés dans le Zou. Les abats de poisson et les résidus de gingembre sont retrouvés dans le département du littoral. De façon globale, les coproduits identifiés peuvent être fournis toute l'année; ils sont donc disponibles pour une production continue des larves de H. illucens. Des trois groupes de coproduits agricoles, les tourteaux sont les plus abondants suivis des résidus et des sons.

Considérant la disponibilité temporelle, 5 coproduits (farine de baobab, épluchures d'igname, résidus de mangue, farine de blé avariée et son de sorgho) sont éliminés en vue de l'élevage industriel des larves de la mouche-soldat noire. En marge de cette sélection, le tourteau d'arachide entre en compétition avec une autre filière, notamment la production de "galettes d'arachide » pour la consommation humaine (30). Ainsi, 22 coproduits sont encore susceptibles d'être utilisés 
Inventaire des coproduits agricoles potentiellement utilisables pour la produ...

pour l'élevage de $H$. illucens. Un autre critère de sélection repose sur la valeur marchande et doit tenir compte de deux facteurs. Premièrement, une étude de marché devra être réalisée afin de déterminer le coût d'un coproduit. Deuxièmement, le but final d'une telle entreprise est de produire des poissons à moindre coût au Bénin. Dès lors, des études de rentabilité économique doivent être effectuées sur une filière de poissons produits à partir des larves de mouche-soldat noire qui ont été nourries avec divers coproduits identifiés. Dans l'optique de produire des larves nutritionnellement enrichies pour satisfaire les besoins du poisson, plusieurs auteurs ont démontré que la composition des larves de $H$. illucens en protéines et lipides est intimement liée à celle de leur régime alimentaire $(22,31)$.

Des premiers essais d'élevage de $H$. illucens ont été réalisés dans cette étude sur trois coproduits (tourteau de coton, son de maïs et abats de poisson) d'intérêt issus chacun d'un des trois groupes identifiés. Parmi les sons les plus disponibles au Bénin (son de maïs et son de blé), le son de blé ayant déjà été testé dans d'autres études $(5,17)$ et possédant un coût plus important, le choix de cette étude s'est porté sur le son de maïs. Des deux tourteaux majoritairement produits, le tourteau de coton est moins couteux que celui du soja et le tourteau de soja est déjà utilisé en alimentation du poisson (6). Dans le groupe des résidus, les abats de poissons ont été retenus dans la mesure où ils sont obtenus gratuitement. Par ailleurs ils présentent un avantage nutritionnel avéré dans l'optimisation de la qualité lipidique chez la mouche-soldat noire (32).

$\mathrm{Au}$ plan nutritionnel, les teneurs en protéines des prépupes sont bien supérieures à celles des régimes, ce qui témoigne d'un fort potentiel de valorisation de coproduits locaux. Les teneurs en protéines $(47,89 \pm 0,11 \%$ et $47,10 \pm 0,11 \%)$ des prépupes issues respectivement des régimes expérimentaux R1 (son de maïs + abats de poisson) et R2 (son de maïs + tourteau de coton) permettent d'envisager un remplacement de la farine de poisson pour couvrir les besoins protéiques du tilapia du Nil qui s'élèvent à $30 \%$ (21). Ces teneurs restent dans la même gamme $(46,30$ \%) que celles obtenues par Oonincx et al. (22) pour des prépupes élevées à partir de coproduits agricoles. La teneur en protéine $(22,90 \%)$ des substrats formulés était semblable à celle des régimes de cette étude. La composition lipidique des prépupes des substrats expérimentaux $(26,75$ $\%$ et $27,70 \%$ ) permet également une couverture des besoins en lipides totaux estimés à $10 \%$ chez O. niloticus (21). Bien que les diètes expérimentales soient isoprotéiques et isolipidiques, une différence apparaît dans la composition des prépupes ainsi que dans leur croissance suggérant une influence de la nature et de la quantité du coproduit ingéré $(11,20)$. Ainsi les larves élevées avec la provende de poulet atteignent plus vite le stade prépupal avec des poids moyens plus élevés que les autres (0.18 g contre 0,13 g et 0,14 g respectivement pour R1 et R2). Spranghers et al. (31) ont observé les mêmes différences de croissance entre les prépupes de la provende $(0,22 \mathrm{~g})$, des résidus de restaurant $(0,15 \mathrm{~g})$ et des déchets végétaux $(0,14 \mathrm{~g})$. Toutefois, les temps de croissance des prépupes de la présente étude (10, 12 et 16 jours pour R0, R1, R2) sont sensiblement inférieurs à ceux de ces auteurs $(12,30 ; 15,50$ et 19,00 jours respectivement pour la provende, les déchets végétaux et les résidus de restaurant). Les pertes de poids observées après les pics de croissance seraient liées au stade de développement de l'espèce comme démontré par Rachmawati et al. (26) qui ont observé une réduction de la teneur en protéine au cours du développement larvaire et une augmentation de la teneur en matière sèche à l'approche du dernier stade. Des deux régimes à base de coproduits testés, seul celui comportant le tourteau de coton présente un fort taux de mortalité et une faible décomposition du substrat. Une cause plausible serait une bioaccumulation de pesticides utilisés dans les pratiques culturales du coton au Bénin (1). La présence d'un facteur antinutritionnel pourrait également expliquer ces mortalités. Le gossypol, une substance 
très toxique contenue dans le coton est connue pour assurer une fonction de défense contre les insectes et les herbivores (18). Sur la base des analyses de cette expérience, le régime R1 favorise un élevage optimal des larves de mouche-soldat noire à partir des coproduits utilisés. Le tourteau de coton tel qu'obtenu dans les structures de production n'est pas recommandé pour l'élevage de cette mouche.

La méthode d'échantillonnage dans le cadre de cette enquête limite la détection de certaines ressources d'intérêts. En effet des essais d'élevage des larves de $H$. illucens ont été réalisées avec des plantes fourragères ou des adventices (2). Ces filières pourraient être investiguées dans le cadre d'une étude qui cible les zones agroécologiques et les élevages de ruminants.

\section{Conclusion}

Cette étude a permis d'inventorier une large gamme de coproduits agricoles dont les tourteaux sont les plus abondants. Parmi ces coproduits, 22 ont été retenus pour servir de matières de base dans les formulations alimentaires destinées à la production des larves d'Hermetia illucens. Globalement, les coproduits sont bien diversifiés et distribués dans les 8 départements enquêtés. Ils sont également disponibles et accessibles tout au long de l'année. L'essai d'élevage réalisé a permis de produire des prépupes dont les compositions nutritionnelles permettent d'envisager un remplacement de la farine de poisson par celle de la mouche-soldat noire dans l'aliment de O. niloticus au Bénin. D’autres expériences pourraient être conduites à l'avenir à partir de la combinaison d’autres coproduits de la sélection pour une évaluation plus poussée.

\section{Remerciements}

Nos sincères remerciements vont à l'Académie de Recherche et d'Enseignement Supérieur (ARES) pour avoir financé la réalisation de ces travaux dans le cadre du projet de développement intitulé "Optimisation de la filière tilapia par l'amélioration génétique et les stratégies alimentaires innovantes" au Bénin pour la période de 2017 à 2021.

\section{Références bibliographiques}

1. Caruso D., Devic E, Subamia I.W., Talamond P. \& Baras E., 2014, Technical handbook of domestication and production of diptera Black Soldier Fly (BSF) Hermetia illucens, Stratiomyidae. IPB Press, Bogor, Indonesia, 141pp

2. Chikou A., 2006, Etude de la démographie et de l'exploitation halieutique de six espèces de poissons-chats (Teleostei, Siluriformes) dans le delta de l'Ouémé au Bénin. Thèse de doctorat : Laboratoire de Démographie des Poissons et d’Hydroécologie, Université de Liège (Belgique).

3. Čičková H., Newton G.L, Lacy R.C. \& Kozánek M., 2015, The use of fly larvae for organic waste treatment. Waste Management, 35, 68-80. https://doi.org/10.1016/j.wasman.2014.09.026

4. Clariza Samayoa A., Chen W.T., \& Hwang S.Y, 2016, Survival and Development of Hermetia illucens (Diptera: Stratiomyidae): A Biodegradation Agent of Organic Waste. Journal of Economic Entomology, 109(6), 2580-2585. https://doi.org/10.1093/jee/tow201 
Inventaire des coproduits agricoles potentiellement utilisables pour la produ...

5. Dersjant-li Y, 2002, The use of soy protein in aquafeeds. In: Cruz-Suárez L.E., Ricque-Marie D., Tapia-Salazar M., Gaxiola-Cortés M.G., Simoes N. (Eds.). Avances en Nutrición Acuícola VI. Memorias del VI Simposium Internacional de Nutrición Acuícola. 3 al 6 de Septiembre del 2002. Cancún, Quintana Roo, México.

6. Diener S., Zurbrugg C., Roa Gutiérrez F., Nguyen H. D., Morel A., Koottatep T., \& Tockner K., 2011, Black soldier fly larvae for organic waste treatment - prospects and constraints. In: Alamgir M., Bari Q.H., Rafizul I.M., Islam S.M.T., Sarkar G. \& Howlader M.K., eds.* Solid Waste Management in Developing Countries. Proceedings of WasteSafe 2011 2nd International Conference, on 13-15 February 2011, Khulna Bangladesh*, 978-984.

7. El Ayoubi H. \& Failler P., 2013, Industrie des pêches et de l'aquaculture au Bénin. Rapport Technique $\mathrm{n}^{\circ} 5$.

8. FAO, 2018, La situation mondiale des pêches et de l'aquaculture. Atteindre les objectifs de développement durable. Rome.

9. Fiogbe E., Akitikpa B. \& Accodji J.M.M., 2009, Essais de mise au point de formules alimentaires à base d'azolla (Azolla microphylla kaulf) et de sous-produits locaux pour la pisciculture rurale du tilapia Oreochromis niloticus L. International Journal of Biological and Chemical Sciences, 3, 2, 398-405. https://doi.org/10.4314/ijbcs.v3i2.44511

10. Gobbi P., Martínez-Sánchez A. \& Rojo S., 2013, The effects of larval diet on adult lifehistory traits of the black soldier fly, Hermetia illucens (Diptera: Stratiomyidae). European Journal of Entomology, 110, 3, 461-468. https://doi.org/10.14411/eje.2013.061

11. Henry M., Gasco L., Piccolo G. \& Fountoulaki E., 2015, Review on the use of insects in the diet of farmed fish: Past and future. Animal Feed Science and Technology, 203, 1, 1-22. https://doi.org/10.1016/j.anifeedsci.2015.03.001

12. Janssen R. H., Vincken J.P., van den Broek L.A.M., Fogliano V. \& Lakemond C.M.M., 2017, Nitrogen-to-Protein Conversion Factors for Three Edible Insects: Tenebrio molitor , Alphitobius diaperinus, and Hermetia illucens. Journal of Agricultural and Food Chemistry, 65(11), 2275-2278. https://doi.org/10.1021/acs.jafc.7b00471

13. Lederoun D., Chikou A., Vreven E., Snoeks J., Moreau J., Vandewalle P. \& Lalèyè P., 2015, Population parameters and exploitation rate of Sarotherodon melanotheron melanotheron Rüppell, 1852 (Cichlidae) in Lake Toho, Benin. Journal of Biodiversity and Environmental Sciences, 6, 2, 259-271.

14. Lederoun D., Vandewalle P., Brahim A., Moreau J. \& Lalèyè P., 2016, Population parameters and exploitation rate of Sarotherodon galilaeus galilaeus (Cichlidae) in Lakes Doukon and Togbadji, Benin. African Journal of Aquatic Science, 41, 2, 151-160. https://doi.org/ $10.2989 / 16085914.2016 .1169988$

15. Makkar H.P.S., Tran G., Heuzé V. \& Ankers P., 2014, State-of-the-art on use of insects as animal feed. Animal Feed Science and Technology, 197, 1-33. https://doi.org/10.1016/ j.anifeedsci.2014.07.008

16. Maurer V., Holinger M., Amsler Z., Früh B., Wohlfahrt J., Stamer A. \& Leiber F., 2016, 
Replacement of soybean cake by Hermetia illucens meal in diets for layers. Journal of Insects as Food and Feed, 2(2), 83-90. https://doi.org/10.3920/JIFF2015.0071

17. Médale, F., \& Kaushik, S. (2009). Les sources protéiques dans les aliments pour les poissons d'élevage. Cahiers Agricultures, 18(2), 103-111. https://doi.org/10.1684/agr.2009.0279

18. Nguyen T.T.X., Tomberlin J. K. \& Vanlaerhoven S., 2013, Influence of Resources on Hermetia illucens (Diptera: Stratiomyidae) Larval Development. Journal of Medical Entomology, 50, 4, 898-906. https://doi.org/10.1603/ME12260

19. Nguyen T.T.X., Tomberlin J. K. \& Vanlaerhoven S., 2015, Ability of Black Soldier Fly (Diptera: Stratiomyidae) Larvae to Recycle Food Waste. Environmental Entomology, 44(2), 406-410. https://doi.org/10.1093/ee/nvv002

20. NRC.,1993. Nutrient Requirements of Fish (National A). https://doi.org/10.1016/ 0022-0981(94)90094-9

21. Oonincx D.G.A.B., Van Broekhoven S., Van Huis A. \& Van Loon J.J.A., 2015, Feed conversion, survival and development, and composition of four insect species on diets composed of food by-products. PLoS ONE, 10, 12, e0144601. https://doi.org/10.1371/journal.pone.0144601

22. Parra Paz A.S., Carrejo N.S. \& Gómez Rodríguez C.H., 2015, Effects of Larval Density and Feeding Rates on the Bioconversion of Vegetable Waste Using Black Soldier Fly Larvae Hermetia illucens (L.), (Diptera: Stratiomyidae). Waste and Biomass Valorization, 6, 6, 1059-1065. https://doi.org/10.1007/s12649-015-9418-8

23. Paul A., Frederich, M., Uyttenbroeck, R., Filocco, S., Hatt, S., Malik, P., Monty A., Francis F., Blecker C. \& Danthine S., 2015, Proximate analysis of seeds from some field. Scientific Bulletin. Series F. Biotechnologies, XIX, 354-359.

24. PROVAC, 2012, Projet de vulgarisation de l'aquaculture continentale en République du Bénin. Lettre d'information Du PROVAC, 2.

25. Rachmawati R., Buchori D., Hidayat P., Hem S., \& Fahmi M.R., 2015, Perkembangan dan Kandungan Nutrisi Larva Hermetia illucens (Linnaeus) (Diptera: Stratiomyidae) pada Bungkil Kelapa Sawit. Jurnal Entomologi Indonesia, 7(1), 28. https://doi.org/10.5994/ jei.7.1.28

26. Rurangwa E., van den Berg J., Lalèyè P., van Duijn A. \& Rothuis A., 2014, Mission exploratoire: Pêche, Pisciculture et Aquaculture au Bénin. Un Quick Scan Du Secteur Pour Des Possibilités d'interventions, 27.

27. Sheppard D.C., Tomberlin J.K., Joyce J.A., Kiser B.C. \& Sumner S.M., 2002, Rearing Methods for the Black Soldier Fly (Diptera: Stratiomyidae): Table 1. Journal of Medical Entomology, 39, 4, 695-698. https://doi.org/10.1603/0022-2585-39.4.695

28. Sodjinou E., Mensah G.A. \& Mongbo R.L., 2016, Aliments, ressources alimentaires et pratiques de nourrissage dans les exploitations piscicoles du Sud-Bénin. Document technique d'information $N^{\circ} 8545$.

29. Soule B.G., Bello R., Orou B.M.A. \& Gibigaye M., 2004, Artisanat alimentaire, Sécurité 
Inventaire des coproduits agricoles potentiellement utilisables pour la produ...

alimentaire et Lutte contre la pauvreté en Afrique: le cas du Bénin. Rapport Laboratoire d'analyse regionale et d'expertise sociale.

30. Spranghers T., Ottoboni M., Klootwijk C., Ovyn A., Deboosere S., De Meulenaer B., Michiels J., Eeckhout M., De Clercq P. \& De Smet S., 2017, Nutritional composition of black soldier fly (Hermetia illucens) prepupae reared on different organic waste substrates. Journal of the Science of Food and Agriculture, 97, 8, 2594-2600. https://doi.org/10.1002/jsfa.8081

31. St-Hilaire S., Cranfill K., McGuire M.A., Mosley E.E., Tomberlin J.K., Newton L., Sealey W., Sheppard C. \& Irving S., 2007, Fish Offal Recycling by the Black Soldier Fly Produces a Foodstuff High in Omega-3 Fatty Acids. Journal of the World Aquaculture Society, 38, 2, 309-313. https://doi.org/10.1111/j.1749-7345.2007.00101.x

32. Tomberlin J.K., Sheppard D.C. \& Joyce, J.A., 2006, Selected Life-History Traits of Black Soldier Flies (Diptera: Stratiomyidae) Reared on Three Artificial Diets. Annals of the Entomological Society of America, 95, 3, 379-386. https://doi.org/10.1603/ 0013-8746(2002)095[0379:slhtob]2.0.co;2

PDF généré automatiquement le 2023-04-26 12:49:48

Url de l'article : https://popups.uliege.be/2295-8010/index.php?id=1587 